\title{
Portal Cholangiopathy: An Uncommon Cause of Right Upper Quadrant Pain
}

\author{
Vikram B. Itare $^{1}$, Donya Imanirad ${ }^{2}$, Abdulaziz Almaghraby ${ }^{2}$ \\ 1. Internal Medicine, Smolensk State Medical University, Smolensk, RUS 2. Internal Medicine, University of South \\ Florida, Tampa, USA
}

Corresponding author: Vikram B. Itare, vikramitare@hotmail.com

\begin{abstract}
Portal cholangiopathy is one of the complications of the chronic portal vein thrombosis (PVT). Chronic PVT can occur in a patient with acute PVT that usually does not resolve regardless of the treatment. There is a development of collateral blood vessels that bring blood from the portal system towards the liver around the obstruction area, known as the cavernous transformation of the portal vein or portal cavernoma, in a patient with chronic PVT. The appearance and location of collateral channels depends on the extent and location of thrombus in the portomesenteric venous system. If the portomesenteric venous system is occluded near the formation of the portal vein, blood tends to flow through collateral channels that form varices in and around the common bile duct. Portal cholangiopathy (also referred to as portal biliopathy) is common in patients with long-standing chronic PVT. It is due to compression of the large bile ducts by the venous collaterals that form in patients with chronic PVT. Most of the patients with long-standing PVT have portal cholangiopathy. Typically, symptoms of portal cholangiopathy include jaundice, biliary colic, and pruritus. Portal cholangiopathy is a rare complication of chronic portal hypertension, and it is an important differential diagnosis of biliary colic secondary to cholelithiasis. The patient can also present with the sharp right upper quadrant pain, which is atypical by nature for biliary colic.
\end{abstract}

Received 08/15/2020

Review began 08/23/2020 Review ended 09/05/2020 Published 09/06/2020

\section{(c) Copyright 2020}

Itare et al. This is an open access article distributed under the terms of the Creative Commons Attribution License CC-BY 4.0., which permits unrestricted use, distribution, and reproduction in any medium, provided the original author and source are credited.
Categories: Internal Medicine, Pathology, Gastroenterology

Keywords: portal cavernoma cholangiopathy, ruq pain

\section{Introduction}

Hunt noted the earliest evidence of portal cholangiopathy in 1965 [1]. Meredith et al. and others reported a few cases of common bile duct obstruction caused by the extensive collateral venous circulation of the portal vein $[2,3]$.

Portal cholangiopathy is an obstruction of the biliary tract in association with engorgement of collateral veins in the hepatic hilum [4]. When portal vein thrombosis (PVT) occurs, multiple venous collaterals develop to bypass the obstruction, which can cause extrinsic compression of the bile ducts. Portal cavernoma cholangiopathy consists of extrahepatic portal vein obstruction (EHPVO) and subsequent cavernous transformation of the portal vein. Most cases of EHPVO manifest as an extrinsic indentation on the bile duct and mild bile duct narrowing, but the majority are asymptomatic. However, progressive portal cavernoma cholangiopathy may lead to severe complications, such as secondary biliary cirrhosis.

Chronic PVT can lead to cavernous or variceal changes in the portal vein forming the collateral blood flow around the gallbladder, which usually leads to non-specific or ischemic changes in the gallbladder wall.

In general, the patients are asymptomatic, but jaundice, cholangitis, and biliary lithiasis may be present in $5 \%$ to $38 \%$ of patients [4-9]. Asymptomatic cases are detected by having biliary abnormalities either on endoscopic retrograde cholangiography (ERC) or magnetic resonance cholangiography (MRC) in the absence of any biliary symptoms. We describe a case of a 63-year-old man who presents with right upper quadrant (RUQ) pain for two days, secondary to portal cholangiopathy caused by chronic portal thrombosis and cavernous formation of the portal vein.

\section{Case Presentation}

A 63-year-old male patient with a past medical history of diabetes milieus, hypothyroidism, laryngeal cancer, hyperlipidemia, carotid artery stenosis, and a previous episode of pancreatitis presented to the emergency room (ER) with the RUQ pain. This pain bothered him for two days and woke him up from sleep. He characterized the pain as sharp, constant, waxing, and waning in intensity, worse with movement, and alleviated by staying still. He denied taking any medicine at home for pain control. The patient did not have nausea, vomiting, fever, or chills. He also denied any history of chronic non-steroidal antiinflammatory drug (NSAID) or alcohol use. On physical examination, the patient had severe RUQ pain on superficial palpation but a negative Murphy sign. 


\section{Cureus}

CT scan showed non-specific gallbladder wall edema, possibly related to the cavernous transformation of the portal vein around the pericholecystic area (Figure 1).

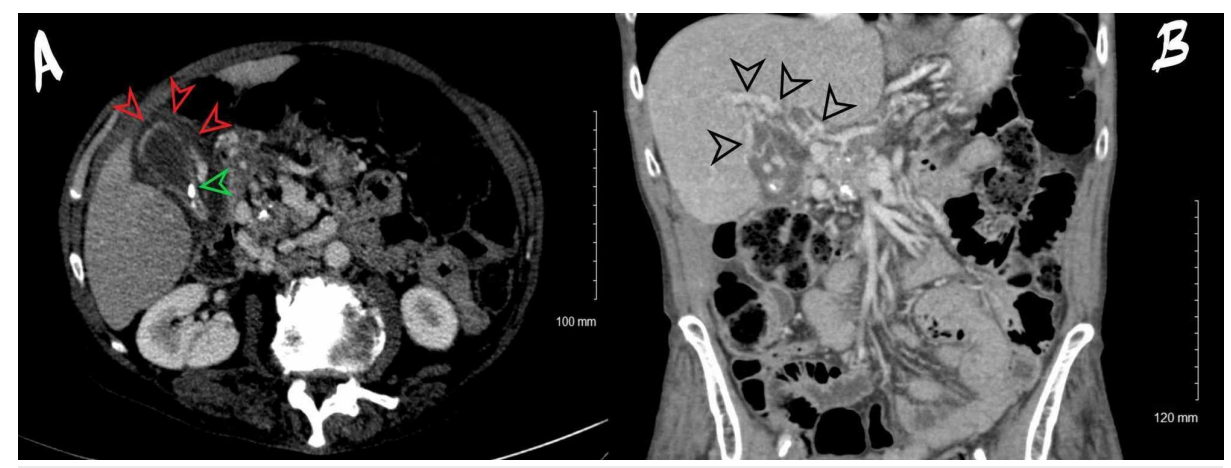

\section{FIGURE 1: CT scan.}

(A) Axial CT scan through the gallbladder; gallbladder: two gallstones are present (green arrow). The gallbladder is not overly distended. There is pericholecystic fluid present (red arrows); pancreas: the pancreas is atrophic with parenchymal calcifications and prominence of the pancreatic duct. (B) Coronal reconstruction CT scan through porta hepatis shows normal caliber bile ducts. There is a cavernous transformation of the portal vein (black arrow). Numerous varices are identified, including perigastric and pericholecystic.

Hepatobiliary iminodiacetic acid (HIDA) scan showed no cystic or common duct obstruction. The gallbladder was visualized after 60 minutes and after morphine administration as above, findings suggestive of chronic gallbladder disease (Figure 2).

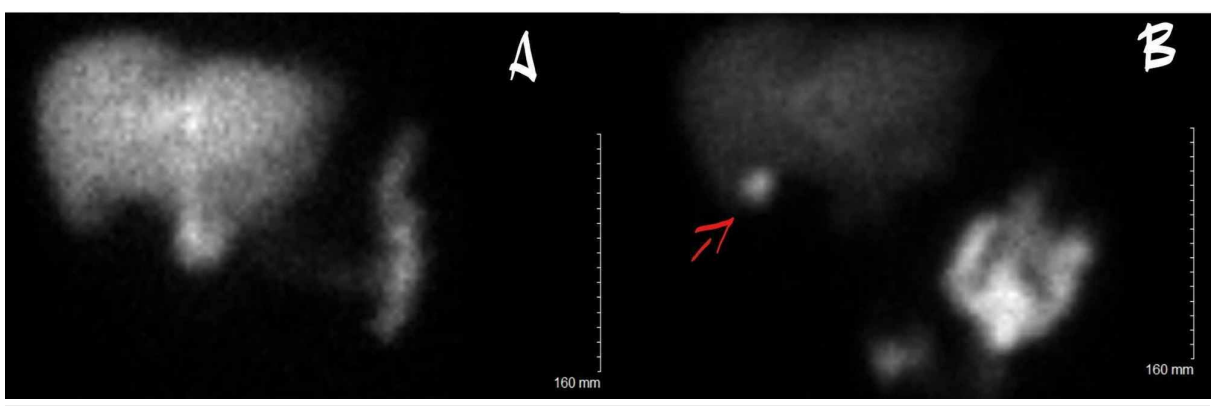

FIGURE 2: Hepatobiliary iminodiacetic acid (HIDA) scan

The initial radioangiogram shows an excellent flow to the liver (not pictured).

(A) Dynamic images show a uniform distribution of the tracer in the liver - excretion in the gut present by 15 minutes. The gallbladder is not visualized 60 minutes into the study. (B) At this time, additional $1.1 \mathrm{mCi}$ of technetium $99 \mathrm{~m}$ choletec is administered. Approximately $2 \mathrm{mg}$ of morphine sulfate is given intravenously, and imaging is continued. The gallbladder is visualized following morphine administration (red arrow).

Impression: No cystic or common duct obstruction. The gallbladder is visualized (red arrow) after 60 minutes and after morphine administration as above, findings suggestive of chronic gallbladder disease.

Laboratory investigations are summarized in Table 1 that revealed the initial derangement in the hepatic profile, which showed subsequent improvement. 


\section{Cureus}

\begin{tabular}{|l|l|l|l|l|l|}
\hline Days & 1 & 2 & 3 & 4 & 5 \\
\hline Aspartate aminotransferase & 31 & $166(\mathrm{H})$ & $60(\mathrm{H})$ & 44 & 30 \\
\hline Alanine aminotransferase & 43 & $251(\mathrm{H})$ & $153(\mathrm{H})$ & $104(\mathrm{H})$ & $76(\mathrm{H})$ \\
Alkaline phosphatase & 77 & $294(\mathrm{H})$ & $237(\mathrm{H})$ & $221(\mathrm{H})$ & $201(\mathrm{H})$ \\
\hline
\end{tabular}

TABLE 1: Lab results.

Subsequently, according to the diagnostic results, it was recommended against any operative procedures like cholecystectomy because of the high risk of hemorrhage due to collateral portal cavernoma formation, which is highly vascularized in the pericholecystic region, secondary to chronic PVT.

After a discussion of the risk of bleeding, it recommended starting anticoagulation with direct oral anticoagulation, apixaban, indefinitely as long as there are no severe bleeding issues to prevent recurrent thrombosis, thrombus extension, and development of portal hypertension.

Lastly, the patient was started on propranolol for the prevention of gastric variceal bleeding due to portal venous hypertension, which developed secondary to the chronic portal thrombosis. Propranolol will reduce the risk of variceal bleeding, which is the most common presenting feature of chronic portal hypertension.

Finally, the diagnosis of portal cholangiopathy was established based on CT scan findings showing gallbladder wall edema, possibly related to the cavernous transformation of portal vein around the pericholecystic area. Usually, the patients presenting with jaundice, recurrent biliary symptoms secondary to portal cholangiopathy, may benefit from endoscopic removal of bile duct stone followed by biliary stent insertion or creation of TIPS (transjugular intrahepatic portosystemic shunt). As this patient presented with the first episode of RUQ pain lasting for two days, with the concern of bleeding, all the surgical procedures were withheld. Our treatment approach followed using apixaban and propranolol.

\section{Discussion}

Portal cholangiopathy is secondary to the cavernous formation of the portal vein in the pericholecystic region, causing the changes in the gallbladder wall. Cavernous or variceal changes in the portal vein are usually due to an increase in pressure and formation of collateral in the portal vein due to an increase in pressure and decrease in portal vein flow towards the liver secondary to the chronic PVT.

Biliary changes are present in about $77 \%-100 \%$ of patients with portal cholangiopathy $[6,7,10]$; however, only $5 \%-38 \%$ of patients developed biliary symptoms $[7,10]$. Symptoms and clinical manifestations of portal cholangiopathy can be related to chronic cholestasis or biliary stone formations, and they include jaundice, cholangitis, cholecystitis, abdominal pain, and cholelithiasis [11].

Risk factors for symptom occurrence in portal cholangiopathy are older age, longer duration of disease, common bile duct, gallbladder stones, and abnormal liver function tests (LFTs) [12].

In biliary colic, pain is neither exacerbated by movement nor relieved by squatting bowel movements or passage of flatus [13]. The pain typically lasts at least 30 minutes, plateauing within an hour. The pain then starts to subside, with an entire attack usually lasting less than six hours [14].

Most patients with chronic PVT are asymptomatic, and the diagnosis is identified incidentally by imaging done for other reasons. Patients with chronic PVT frequently have esophageal or gastric varices, and the most common clinical presentation is gastrointestinal bleeding [15]. In a retrospective series that included 40 patients with chronic newly diagnosed PVT, esophageal varices were present in 35 patients (88\%), gastric varices in 20 patients (50\%), portal hypertensive gastropathy in 19 patients (48\%), and gastrointestinal bleeding in 19 patients (48\%).

In summary, portal cholangiopathy is a rare complication of chronic portal hypertension, and it is a crucial differential diagnosis of biliary colic. Nevertheless, in this case, the patient presented with atypical features of biliary colic, including RUQ pain, was not post-prandial, which was aggravated due to movement. The duration of the pain was more than 48 hours.

\section{Conclusions}

Jaundice and RUQ pain in this patient prompted the work-up for the cause. Portal cavernoma is an uncommon cause for these findings and can be an unrecognized diagnosis; hence, it should be included 
among the differential diagnoses for extrahepatic biliary obstruction.

Differential diagnosis of any patient with jaundice and RUQ pain should consider hepatic hilum neoplasia, such as cholangiocarcinoma or metastatic neoplasms, as well as other causes of intrahepatic bile ducts involvement, such as sclerosing cholangitis.

The gold standard for diagnosis of portal cavernoma is endoscopic retrograde cholangiopancreatography (ERCP). Doppler ultrasound studies can show gallbladder varices in patients. Patients with suspected portal biliopathy should be evaluated with MRC, and the results should be compared with the results of ERCP studies.

Morphological alterations in cholangiography can range from biliary tract dilatation associated with areas of stenosis and angulations to failure in duct filling due to compression by dilated parietal veins projecting towards the lumen.

Patients with symptoms and recurrent cholangitis can be treated using bile duct dilation. Before bile duct dilatation, portal decompression is recommended.

\section{Additional Information}

\section{Disclosures}

Human subjects: Consent was obtained by all participants in this study. Conflicts of interest: In compliance with the ICMJE uniform disclosure form, all authors declare the following: Payment/services info: All authors have declared that no financial support was received from any organization for the submitted work. Financial relationships: All authors have declared that they have no financial relationships at present or within the previous three years with any organizations that might have an interest in the submitted work. Other relationships: All authors have declared that there are no other relationships or activities that could appear to have influenced the submitted work.

\section{Acknowledgements}

Candice Mateja, DO (Associate Professor, Morsani College of Medicine) for her guidance and support and Michael Lombino, M.D. (Chairman Radiology, Bronxcare Health System) for reading the radiological images.

\section{References}

1. Hunt $\mathrm{AH}$ : Compression of the common bile-duct by an enlarging collateral vein in a case of portal hypertension. Br J Surg. 1965, 52:636-637. 10.1002/bjs.1800520820

2. Meredith HC, Vujic I, Schabel SI, O'Brien PH: Obstructive jaundice caused by cavernous transformation of the portal vein . Br J Radiol. 1978, 51:1011-1012. 10.1259/0007-1285-51-612-1011

3. Spira R, Widrich WC, Keusch KD, Jackson BT, Katzman HE, Coello AA: The bile duct varies . Arch Surg. 1985, 120:1194-1996. 10.1001/archsurg.1985.01390340086018

4. Walser EM, Runyan BR, Heckman MG, et al.: Extrahepatic portal biliopathy: proposed etiology on the basis of anatomic and clinical features. Radiology. 2011, 258:146-153. 10.1148/radiol.10090923

5. Dhiman RK, Behera A, Chawla YK, Dilawari JB, Suri S: Portal hypertensive biliopathy. Gut. 2007, 56:10011008. 10.1136/gut.2006.103606

6. Khuroo MS, Yattoo GN, Zargar SA, Javid G, Dar MY, Khan BA, Boda MI: Biliary abnormalities associated with extrahepatic portal venous obstruction. Hepatology. 1993, 17:807-813. 10.1002/hep.1840170510

7. Dilawari JB, Chawla YK: Pseudosclerosing cholangitis in extrahepatic portal venous obstruction . Gut. 1992, 33:272-276. 10.1136/gut.33.2.272

8. Vibert E, Azoulay D, Aloia T, et al.: Therapeutic strategies in symptomatic portal biliopathy . Ann Surg. 2007, 246:97-104.

9. Llop E, de Juan C, Seijo S, et al.: Portal cholangiopathy: radiological classification and natural history . Gut. 2011, 60:853-860.

10. Condat B, Vilgrain V, Asselah T, et al.: Portal cavernoma-associated cholangiopathy: a clinical and MR cholangiography coupled with MR portography imaging study. Hepatology. 2003, 37:1302-1308.

11. Oo YH, Olliff S, Haydon G, Thorburn D: Symptomatic portal biliopathy: a single-center experience from the UK. Eur J Gastroenterol Hepatol. 2009, 21:206-213. 10.1097/MEG.0b013e3283060ees

12. Dhiman RK, Chawla Y, Duseja A, Chhetri D, Dilawari J: Portal hypertensive biliopathy (PHB) in patients with extrahepatic portal venous obstruction (EHPVO). J Gastroenterol Hepatol. 2006, 21:A504.

13. Festi D, Sottili S, Colecchia A, Attili A, Mazzella G, Roda E, Romano F: Clinical manifestations of gallstone disease: evidence from the multicenter Italian study on cholelithiasis (MICOL). Hepatology. 1999, 30:839846. 10.1002/hep.510300401

14. Diehl AK, Sugarek NJ, Todd KH: Portal vein thrombosis; risk factors, clinical presentation and treatment . Am J Med. 1990, 89:29-33. 10.1016/0002-9343(90)90094-t

15. Meseeha M, Attia M: Esophageal Varices. StatPearls Publishing, Treasure Island, FL; 2020. 